Rev. Elev. Med. vêtt. Pays trop., 1975, 28 (4) : 499-511

\title{
Etude d'une population taurine de race Baoulé en Côte-d'Ivoire
}

\author{
par E. TIDORI $\left({ }^{*}\right)$, H. SERRES $\left({ }^{* *}\right)$, D. RICHARD $\left({ }^{* *}\right)$ et J. AJUZIOGU $\left(^{*}\right)$
}

\section{RESUME}

Les taurins Baoulé, les plus nombreux en Côte-d'Ivoire, sont trypanotolérants. Un troupeau a été élevé et contrôlé à Bouaké (région de savane soudano-guinéenne).

Le Baoulé est une petite race : $100 \mathrm{~cm}$ pour les mâles et $95 \mathrm{~cm}$ pour les femelles, au garrot. Mais la race montre son adaptation au milieu par une excellente fertilité, un âge au premier vêlage très précoce et une bonne résistance aux maladies. Les caractéristiques de la croissance sont étudiées.

La finition soit sur pâturage de Stylosanthes guianensis, soit avec un aliment concentré permet d'obtenir une croissance plus rapide.

Les carcasses sont légères et peu grasses. Les mortalités les plus importantes sont dues à la diarrhée des veaux.

\section{INTRODUCTION}

Dans toute la zone soudano-guinéenne d'Afrique de l'Ouest, la présence de glossines entretient la dissémination de la trypanosomiase. Cette dernière interdit l'élevage de nombreuses espèces animales: chevaux, ânes, chameaux, zébus et même taurins. On rencontre cependant dans ces régions des animaux de type taurin qui résistent à cette maladie et sont pour cela appelés «trypano-tolérants».

Deux types principaux sont à distinguer :

- un type à cornes assez longues, à robe fauve ou brune, de format relativement faible, connu sous le nom de race N'Dama. Son aire est essentiellement localisée en Guinée, mais elle déborde au Sénégal, au sud du Mali et au nord-ouest de la Côte-d'Ivoire;

- un type à cornes courtes, à robe beaucoup plus variable, très fréquemment pie avec

(*) Service de l'Elevage, Bouaké, Côte-d'Ivoire.

(**) I.E.M.V.T, 10, rue Pierre Curie, 94700 Maisons-Alfort.

$(* * *)$ Mission Vétérinaire Française, P.O. Box 1053, Addis-Abeba, Ethiopie. une dominante noire et blanche. Son format est encore plus faible que celui du N'Dama. Ce bétail à courtes cornes est en général localisé plus à l'Est : sud-est du Mali, sud de la Haute-Volta (bœuf Lobi), dans le massif de l'Atakora au Dahomey (race Somba), au nord du Nigéria (Nigerian Shorthorn). au Cameroun (race Namchi et race Bakosi). En outre, une variété plus petite encore, dite race des Lagunes, est élevée le long de la côte du Golfe de Guinée.

En Côte-d'Ivoire (Régions du Centre et du Nord), ce bétail à courtes cornes a reçu le nom de race Baoulé. Il y représente environ les quatre cinquièmes de l'élevage bovin national, soit environ 300000 têtes. On peut, au passage, noter que des bovins Baoulés ivoiriens ont été introduits en assez grand nombre en Republique Centrafricaine (Région de Bouar) où ils paraissent prospérer.

L'élevage Baoulé en Côte-d'Ivoire est groupé en plusieurs noyaux nettement individualisés :

- Région de Korhogo-Sinematiali, densément peuplée et cultivée, où le bétail appartenant aux cultivateurs Sénoufo est confié à des 
Peuls qui le gardent et traient les vaches;

- Région de Bouna, où les Lobi sont euxmêmes éleveurs;

- Région centrale de Dabakala et de la vallée du Haut Nzi où le bétail, comme à Korhogo, est presque toujours confié aux Peuls;

- Région de Boundiali enfin, où la race est métissée par des apports de sang N'Dama venant de la région d'Odienné voisine, ou par des zébus venant du sud du Mali par la route à bétail entrant en Côte-d'Ivoire à Tingrela.

Le pays Baoulé, centré sur Bouaké, n’a que peu de bovins et les paysans ne sont pas éleveurs. Le nom choisi pour la race n'est donc pas très judicieux.

Les études de ce bétail sont rares: nous signalerons les rapports de AILLERIE (1), les descriptions de DOUTRESSOULLE (2) et l'ouvrage de JOSHI (4) qui contient des chif-
D'une façon générale, on peut dire que les pâturages, très suffisants les premiers temps, sont devenus assez nettement surchargés en 1972, alors que l'effectif s'était accru.

$\mathrm{Au}$ plan climatique, on retiendra que les températures moyennes varient assez peu au cours de l'année, bien qu'on puisse au maximum enregistrer $40^{\circ}$ en mars et au minimum $14^{\circ}$ en décembre.

Température moyenne annuelle: $26,5^{\circ}$.

Température mensuelle maximale : $28,3^{\circ}$.

Température mensuelle minimale : $24,6^{\circ}$.

L'humidité atmosphérique, toujours élevée, varie de 60 p. 100 en janvier à 82 p. 100 en août.

La pluviosité moyenne annuelle s'élève à $1207 \mathrm{~mm}$ avec 95 jours de pluie. Elle se répartit comme suit :

\begin{tabular}{rrrrrrrrrrrrrr}
\hline & Janv. & Fev. & Mars & Av. & Mai & Juin & Juil. & Août & Sept. & Oct. & Nov. & Déc. & Total \\
$\mathrm{mm}$ & 12,2 & 37,5 & 96,7 & 142,9 & 149,8 & 135,9 & 86,0 & 116,4 & 236,0 & 130,1 & 39,2 & 24,5 & 1207 \\
$\mathbf{j}$ & 1,1 & 3,5 & 6,7 & 7,9 & 9,9 & 10,6 & 8,6 & 12,4 & 16,6 & 11,7 & 3,6 & 2,2 & 95 \\
\hline
\end{tabular}

fres douteux car les femelles y ont un format beaucoup plus important que les mâles. Enfin, VERLY (6) a fait d'intéressantes observations sur le format du bétail, notamment sur les femelles adultes.

Dans les pages qui suivent, nous exposerons les résultats d'observations faites sur un élevage de Baoulés à la station de Bouaké.

\section{MISE EN PLACE DE L'ELEVAGE}

\section{La station de Bouaké}

La station comporte 350 hectares de pâturages naturels qui ont constitué la seule nourriture du bétail. Les sols sont soit argilo-sableux, soit sablo-argileux avec un $\mathrm{pH}$ acide Les graminées de savane que l'on y rencontre comportent des andropogonées qui constituent un bon pâturage (Hyparrhenia chrysargyrea, Hypertalia dissoluta). Mais lorsque la surcharge se manifeste, elles régressent devant des graminées plus dures (Panicum phragmitö̈des) ou des annuelles (Digitaria horizontalis dans les zones basses), alors que l'on voit se multiplier les rejets ligneux (Lophira lanceolata, Daniellia oliveri).
La saison sèche couvre les mois de novembre, décembre, janvier et février et se termine à la mi-mars. Si l'on considère qu'au début de novembre, avant que l'harmattan ne dessèche l'atmosphère, l'humidité du sol est encore suffisante pour s'entretenir par quelques averses, on peut considérer que la période de vie active des plantes est voisine de 8 mois.

Entre le début de juillet et la mi-août, se situe une succession de périodes de cinq à six jours sans pluie et l'on peut même observer quinze jours secs consécutifs. C'est l'amorce du dédoublement de la saison des pluies qui s'accentue vers le Sud. Mais cette petite saison sèche, si elle ralentit le rythme de végétation n'a pas de conséquence sur les conditions de pâturage qui sont donc à considérer comme bonnes.

\section{Constitution du troupeau}

Le troupeau a été constitué en 1967 par l'achat, dans la région de Dabakala, de 40 vaches, 70 génisses et 4 taureaux.

Les animaux ont été choisis bien dans le type, dans des troupeaux homogènes où l'on n'a jamais introduit de sang extérieur. La plupart des animaux sont de robe pie noire. 
Ce troupeau s'est multiplié à la station et quelques introductions complémentaires ont été faites en petit nombre dans les années suivantes. L'effectif des vaches a évolué de la façon suivante :

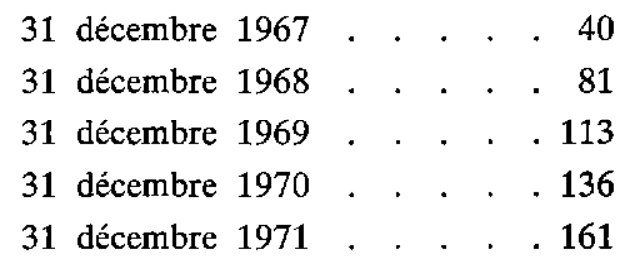

\section{Mode d'élevage}

L'élevage est semi-extensif. Les animaux sont au pâturage toute la journée et le soir rentrés dans un parc, ce qui, en permettant une meilleure surveillance du proupeau, entretient la docilité naturelle du Baoulé.

Les vaches et leurs veaux sont dans un parc séparé, les génisses dans un second, les taurillons et taureaux au repos dans un troisième.

Les veaux sont vermifugés à 15 jours contre l'ascaridiose puis revermifugés tous les deux mois contre les strongles.

L'ensemble du troupeau est annuellement vacciné contre la péripneumonie, la peste bovine, la pasteurellose. Aucun déparasitage externe n'est pratiqué. Aucune complémentation alimentaire n'est jamais distribuée.

\section{CARACTERISTIQUES DE LA RACE BAOULE}

\section{Extérieur des animaux}

Les taurins Baoulés apparaissent d'une façon générale comme des animaux de format réduit, mais d'allure générale assez équilibrée, près de terre mais sans excès.

La tête est de profil rectiligne. Le front, large et plat chez le mâle, présente un léger resserrement chez la femelle entre les cornes et les orbites. Ces dernières sont assez saillantes, ce qui détermine une légère dépression entre elles. Le chanfrein, droit, se termine par un mufle généralement noir et par une bouche plutôt grande. Les oreilles, petites sont portées latéralement et plutôt vers le haut.

Les cornes ont une base forte chez le taureau et se développent de façon conique latéra- lement, pour former un léger croissant chez les mâles âgés. Chez la femelle, le cornage est nettement plus fin et après un démarrage latéral, il se développe obliquement vers l'avant et le haut.

L'encolure, un peu courte, légère chez la vache, plus épaisse chez le mâle, est portée horizontalement; sa ligne supérieure prolonge celle du dos sans discontinuité.

Le garrot se détache peu et la ligne du dos se montre rectiligne et presque horizontale (le sacrum n'est que de très peu plus haut que le garrot: 2 à $3 \mathrm{~cm}$ ).

La côte ronde donne une poitrine ample et le tronc, dans sa partie postérieure, conserve une section cylindrique, les animaux étant rarement ventrus. La mamelle, toujours petite, est remontée entre les cuisses, peu visible latéralement.

Le bassin, peu incliné, présente une pointe de la fesse située légèrement plus bas que l'angle de la hanche; assez long, et assez large au niveau des hanches, il se montre très resserré aux ischiums, ce qui donne un arrière étroit. La queue est attachée un peu haut et sa base se détache nettement au-dessus du bassin. Sa longueur moyenne amène l'extrémité du toupillon au-dessous du jarret, en général à midistance entre le jarret et le sol.

Les membres et les articulations sont fins. L'épaule plutôt verticale se fond bien avec le thorax. La cuisse a le plus souvent un profil rectiligne et ne devient convexe que chez les animaux en excellent état d'embonpoint. Les onglons, petits et noirs, sont formés d'une corne résistante.

Le poil est toujours court, fin et luisant sur les animaux en bonne santé. La robe la plus fréquente est pie noire, soit avec de grandes taches irrégulièrement réparties, soit avec de petites taches noires sur un fond à dominante blanche. On peut trouver des animaux entièrement noirs ou encore des robes fauves ou fauvepie. En somme, la couleur est assez variable et ne peut pas permettre de caractériser la race.

\section{Format des animaux adultes}

Le format des animaux adultes peut être apprécié par les mensurations principales que nous regroupons au tableau $n^{\circ} I$ : 


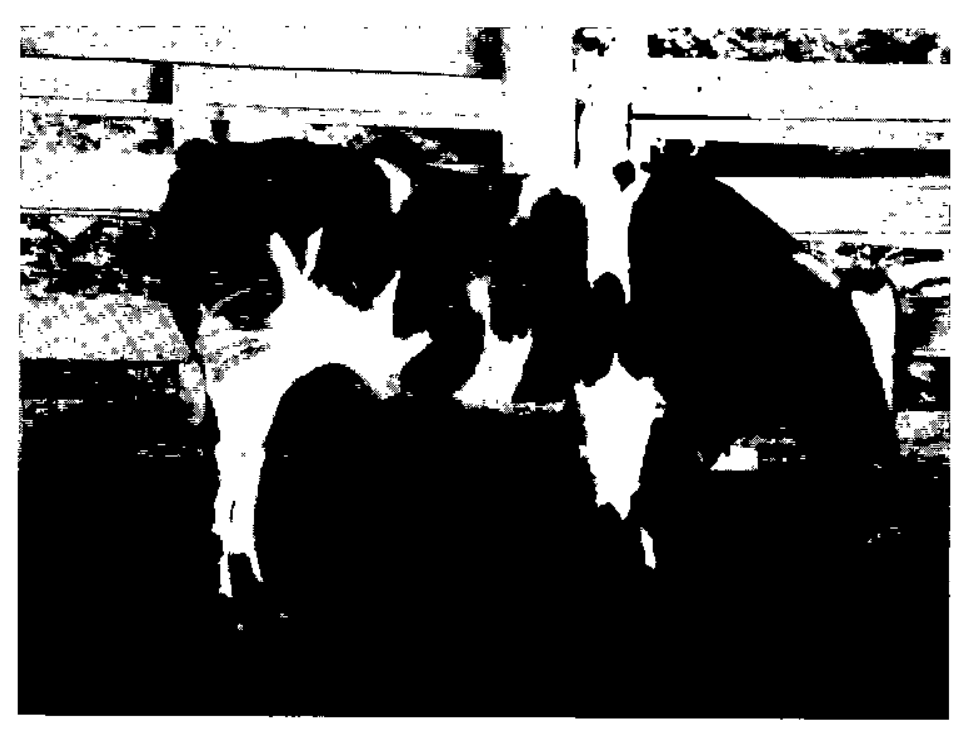

Photo $n^{\circ} 1$

Taureau Baoulé
Photo $\mathrm{n}^{\circ} 2$

Vache Baoulé

en savane
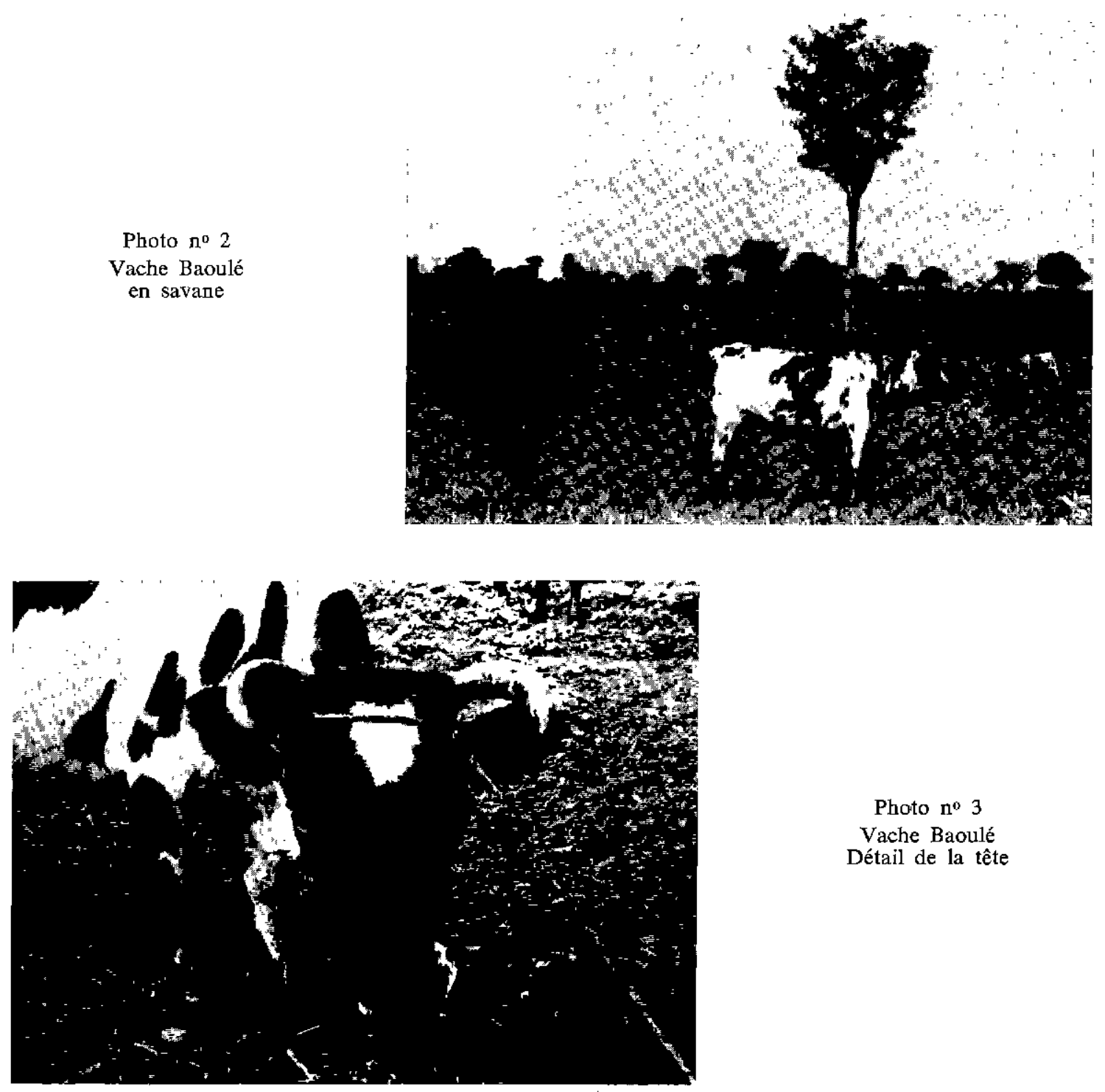

Photo no 3

Vache Baoulé

Détail de la tête 


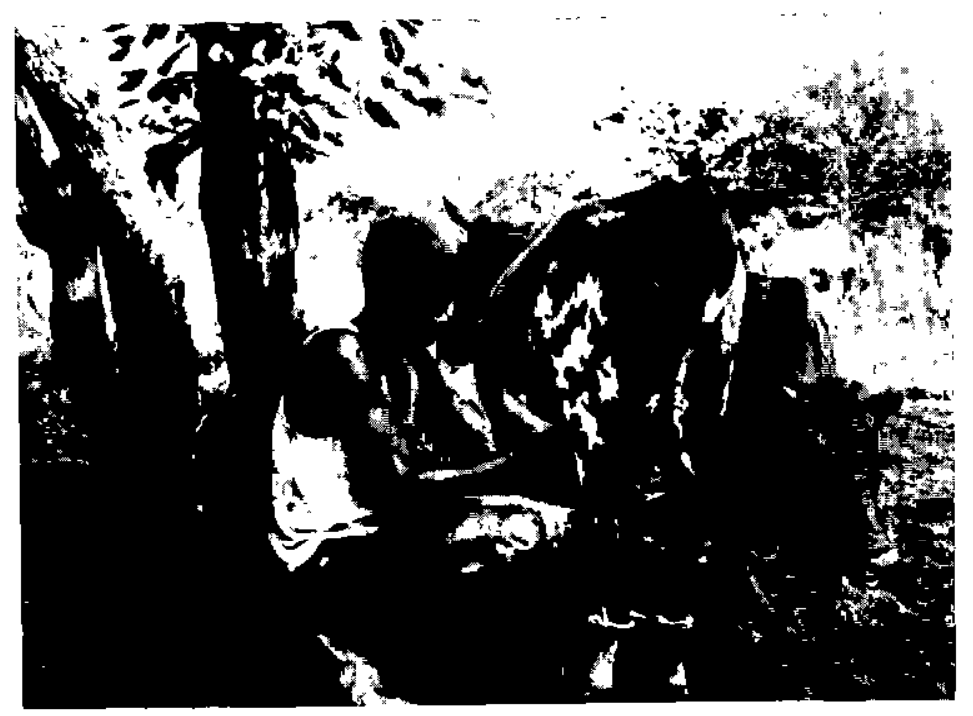

Photo n. 4

Traite par un berger Peul
Photo no 5

Troupeau villageois
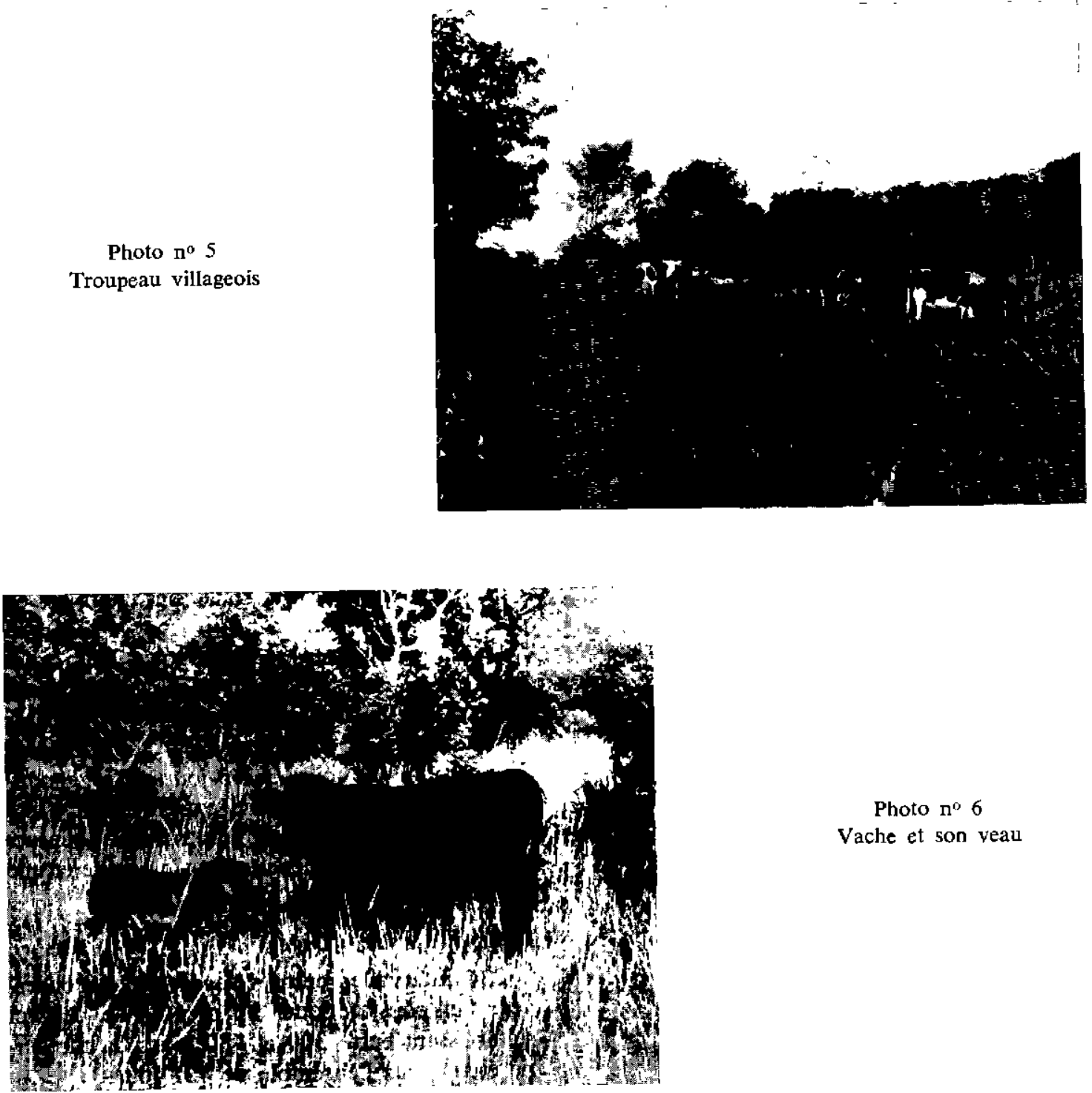

Photo $\mathrm{n}^{\circ} 6$

Vache et son yeau 
TABLEAU $\mathrm{N}^{\mathbf{D}} \mathrm{I}$

\begin{tabular}{|c|c|c|}
\hline & $\begin{array}{l}\text { Males } \\
\mathrm{n}=26\end{array}$ & $\begin{array}{c}\text { Femelles } \\
n=40\end{array}$ \\
\hline Hauteur au garrot $(\mathrm{cm})$ & $100,07 \pm 1,68$ & $95,15 \pm 1,14$ \\
\hline $\begin{array}{l}\text { Hauteur sous-sternale } \\
(\mathrm{cm})\end{array}$ & $48,54 \pm 0,96$ & $46,75 \pm 0,61$ \\
\hline $\begin{array}{l}\text { Périmētre thoracique } \\
\text { (cm) }\end{array}$ & $140,46 \pm 2,60$ & $28,44 \pm 1,6$ \\
\hline $\begin{array}{l}\text { Longueur scapulo- } \\
\text { ischiale }(\mathrm{cm})\end{array}$ & $121,19 \pm 2,58$ & $112,29 \pm 1,7$ \\
\hline $\begin{array}{l}\text { Longueur de la } \\
\text { croupe (cm) }\end{array}$ & $41,15 \pm 0,89$ & $38,00 \pm 0,75$ \\
\hline $\begin{array}{l}\text { Largeur aux hanches } \\
(\mathrm{cm})\end{array}$ & $33,27 \pm 0,77$ & $31,41 \pm 0,66$ \\
\hline
\end{tabular}

Ces chiffres nous montrent bien le petit format de la race Baoulé, sans doute l'un des plus faibles pour des bovins (à l'exception de la race des Lagunes).

Les mensurations moyennes du troupeau de Bouaké peuvent être utilement comparées à celles relevées par VERLY (6), dans diverses régions de Côte-d'Ivoire, sur des vaches Baoulé.
Le premier rapport (P.T./H.G.) donne des chiffres peu élevés : le Baoulé n'est pas un animal compact. Les races « à viande » donnent des rapports nettement plus forts: Charolais mâle 1,70, Limousin mâle 1,69, Charolais femelle 1,53 , Limousin femelle 1,47 .

Le rapport L.S.I./H.G. qui détermine le profil général de l'animal est bon, puisque pour un animal plutôt long, comme le Limousin, on a 1,23 chez le mâle et $1,21 \mathrm{chez}$ la femelle. Le Baoulé doit être qualifié de médioligne, mais non de bréviligne.

En revanche, le rapport de la largeur aux hanches à la longueur de la croupe est faible, car sur les races taurines améliorées, il dépasse toujours 1 (largeur supérieure à la longueur, alors que c'est nettement l'inverse ici). Cela confirme l'aspect étroit de l'arrière train du Baoulé.

Dabakala $(\mathrm{n}=11)$ Bouna $(\mathrm{n}=105)$
Hauteur au garrot $(\mathrm{cm})$
$92,00 \pm 1$
$94,2 \pm 0,3$
Périmètre thoracique $(\mathrm{cm})$.
$131,8 \pm 1,4$
$132,6 \pm 0,5$
Largeur aux hanches (cm) .
$32,00 \pm 0,4$
$33,4 \pm 0,1$

On note une très bonne concordance de ces moyennes avec celles observées sur le troupeau de Bouaké. Si l'on tient compte du facteur de variation lié à la façon de l'observateur de prendre les mesures, on est amené à conclure que le bétail Baoulé est homogène dans les diverses régions considérées et que l'échantillon de Bouaké est bien représentatif de la race.

A partir des mensurations, on peut établir quelques rapports capables de nous aider à caractériser la race.

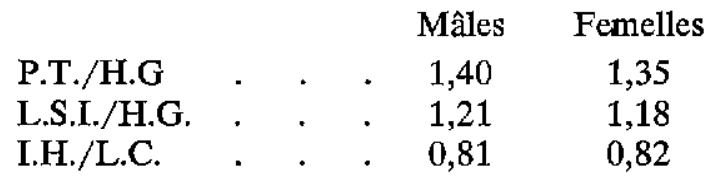

P.T. = Périmètre thoracique.

H.G. = Hauteur au garrot.

L.S.I. = Longueur scapulo-ischiale.

I.H. = Largeur des hanches.

L.C. = Longueur de la croupe.

\section{PRODUCTIVITE DE LA RACE BAOULE}

\section{Reproduction}

\section{Age moyen au premier vêlage}

Les génisses, đès le sevrage, ont été mises en présence du taureau, ce qui permet la fécondation dès qu'elles en sont capables. Sur celles qui sont nées à la station et dont on connaît la idate de naissance, 63 vêlages primipares ont été observés. L'âge moyen s'établit alors à 2 ans 1 mois 21 jours \pm 40 jours. C'est incontestablement une moyenne extrêmement basse pour un bétail élevé en milieu tropical. Il caractérise une grande précocité physiologique et une parfaite adaptation au milieu.

\section{Intervalle entre vêlages}

234 intervalles entre vêlages ont été observés. On constate que 80 p. 100 de ceux-ci s'établissent entre 300 et 480 jours, la moyenne étant pour l'ensemble de 421 jours. 
Cela correspond pour les femelles reproductrices à une fécondité supérieure à 85 p. 100 , vraiment excellente dans des conditions d'élevage pratiquement extensif.

La confirmation est donnée par le nombre de veaux et velles nés chaque année, rapporté au nombre de vaches présentes:

\begin{tabular}{|c|c|c|c|c|}
\hline & 1968 & 1969 & 1970 & 1971 \\
\hline $\mathrm{acl}$ & 81 & 113 & 136 & 161 \\
\hline 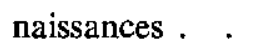 & 68 & 95 & 114 & 120 \\
\hline 100 & 83,95 & 84,07 & 83,82 & 74,53 \\
\hline
\end{tabular}

Le taux de fécondité ne peut pas être donné car les génisses sont mises dans le troupeau à saillir dès l'âge de 9 mois, alors qu'elles ne sont pas encore pubères et la détermination du nombre de femelles aptes à être fécondées ne peut être qu'arbitraire.

\section{Rythme saisonnier des naissances}

Les cycles sexuels des vaches Baoulé sont fortement influencés par les phénomènes saisonniers. 399 naissances observées pendant une période de 4 années, se répartissent comme suit :
On peut invoquer soit le climat sec qui favorise un meilleur état général des animaux, soit une insolation beaucoup plus forte qui entraînerait un meilleur fonctionnement neuro-hormonal.

On constate un léger regain de la courbe vers mars-avril qui correspond à des saillies de juin et juillet.

En revanche, la natalité s'effondre en mai, juin, juillet, ce qui indique une fertilité très faible d'août à octobre, pendant la seconde moitié de la saison des pluies, au moment où ces dernières sont les plus intenses. Le rôle favorable que l'on attribue à l'équinoxe d'automne ne paraît jouer nullement.

Le poids des veaux à la naissance varie dans le sens inverse de la fréquence des naissances : ceux nés en juin, juillet, août, septembre paraissent un peu plus lourds. Cela s'explique car la fin de gestation correspond alors au stade de la meilleure valeur alimentaire du pâturage.

\begin{tabular}{lcccccccccccc}
\hline & Janv. & Fév. & Mars & Avril & Mai & Juin & Juil. & Août & Sept. & Oct. & Nov. & Déc. \\
p. 100 & 8,5 & 6,5 & 6,75 & 5,75 & 4,25 & 2,25 & 2,5 & 9,0 & 9,75 & 16,75 & 16 & 11,75 \\
n & 34 & 26 & 27 & 23 & 17 & 9 & 10 & 36 & 39 & 67 & 64 & 47 \\
\hline
\end{tabular}

Ces chiffres reportés sur le graphique $n^{\circ} I$ permettent de tracer une courbe qui montre un pic très net correspondant aux mois de septembre, octobre, novembre, décembre pendant lesquels se situent 55 p. 100 des naissances, consécutives à des saillies fécondantes situées de décembre à mars, pendant la saison sèche.

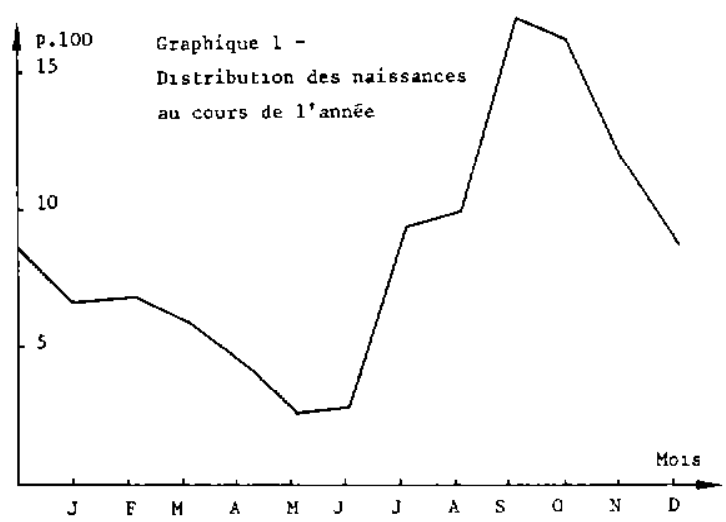

\section{Poids moyens des veaux à la naissance}

$\begin{array}{llll}\text { Janvier } & 11,79 & \text { Juillet } & 14,6 \\ \text { Février } & 12,47 & \text { Août } & 13,08 \\ \text { Mars } & 11,27 & \text { Septembre } & 13,15 \\ \text { Avril } & 12,71 & \text { Octobre } & 12,10 \\ \text { Mai } & 11,86 & \text { Novembre } & 11,18 \\ \text { Juin } & 13,5 & \text { Décembre } & 11,69\end{array}$

\section{Croissance}

La croissance des Baoulés a pu être étudiée par des pesées mensuelles et détermination des poids aux âges types de $3,6,9,12,18$ mois, 2 et 3 ans.

Les poids moyens des mâles et des femelles sont donnés au tableau II.

On peut noter en outre que 3 taureaux de 4 ans pèsent en moyenne $256 \mathrm{~kg}$ et 9 taureaux de 5 ans et plus $267 \mathrm{~kg}$, ce qui peut être considéré comme le poids maximal moyen. 
TABLEAU $N^{\circ} I I-$ Poids à âges types $(\mathrm{kg})$

\begin{tabular}{|l|c|c|c|c|c|c|c|c|}
\hline & Naissance & 3 mois & 6 mois & 9 mois & 12 mois & 18 mois & 2 ans & 3 ans \\
\cline { 2 - 8 } & $12,56 \pm 0,33$ & $36,49 \pm 1,30$ & $61,25 \pm 2,20$ & $61,65 \pm 2,86$ & $92,86 \pm 3,27$ & $127,18 \pm 4,35$ & $162,03 \pm 5,28$ & $212,82+10,06$ \\
& $(176)$ & $(155)$ & $(138)$ & $(123)$ & $(111)$ & $(81)$ & $(65)$ & $(28)$ \\
Fêles & $12,03 \pm 0,25$ & $36,74 \pm 1,05$ & $61,73 \pm 1,92$ & $84,76 \pm 2,31$ & $95,78 \pm 2,72$ & $123,67 \pm 2,83$ & $145,78 \pm 3,37$ & $165,98 \pm 6,42$ \\
& $(184)$ & $(172)$ & $(145)$ & $(144)$ & $(111)$ & $(97)$ & $(41)$ \\
\hline
\end{tabular}

Quant aux vaches adultes, elles pèsent en moyenne $175 \mathrm{~kg}$ (159 observations sur des animaux de 4 ans et plus).

Les gains quotidiens moyens s'établissent comme suit (en $\mathrm{g} / \mathrm{j}$ ) : (Tableau $\mathrm{n}^{\circ}$ III).

TABL, $N^{\circ}$ III-Gains quotidiens moyens ( $\mathrm{g}$ )

\begin{tabular}{|l|c|c|}
\hline \multirow{2}{*}{ Naissance } & Mâles & Femelles \\
\cline { 2 - 3 } 3 mois & 266 & 274,5 \\
6 mois & 275 & 277,6 \\
9 mois & 226,6 & 255,9 \\
12 mois & 124,5 & 122,4 \\
18 mois & 190,6 & 154,9 \\
2 ans & 193,6 & 122,8 \\
3 ans & 139,1 & 55,3 \\
\hline
\end{tabular}

Les mâles et les femelles ont des croissances tout à fait superposables jusqu'à 12 mois. Au cours de cette période, on observe une vitesse de croissance très régulière jusqu'à 6 mois $(275 \mathrm{~g} / \mathrm{j})$ qui fléchit un peu entre 6 et 9 mois, la lactation de la mère devenant faible. Mais un décrochement net se produit après le sevrage à 9 mois, les gains quotidiens diminuant brusquement de moitié. De 1 à 2 ans, on voit les mâles reprendre mieux que les femelles en raison du dimorphisme sexuel. Enfin, après deux ans, alors que les mâles poursuivent une croissance assez active, les femelles voient la leur très ralentie par la première mise bas et le premier allaitement. Le graphique II met parfaitement en évidence ces observations.

Nous ne pouvons pas terminer cette étude de la croissance au pâturage naturel sans souligner l'influence de la saison des naissances.

$\mathrm{Si}$ on distingue les naissances :

I. Décembre, jánvier, février = saison sèche

II. Mars, avril, mai = début des pluies
III. Juin, juillet, août $=$ milieu des pluies

IV. Septembre, octobre, novembre $=$ fin des pluies, on obtient les moyennes de poids, à différents âges, suivantes (kg) :

I. Saison sèche 1 an 18 mois 2 ans

II. Début des pluies

III. Milieu des pluies

$90,15 \quad 133,8$ 152,7 $83,21 \quad 124,18 \quad 149,6$

1V. Fin des pluies 109 119,6 178,6

On voit qu'à 1 an et 2 ans, les animaux les plus lourds sont ceux qui naissent en juin, juillet, août (groupe III); cela s'explique aisément, ces anniversaires venant en saison des pluies où l'herbe nutritive abonde. A l'inverse, ces anniversaires viennent en fin de saison sèche pour le groupe II (mars, avril, mai) et les animaux y sont moins lourds, les deux autres groupes étant intermédiaires. A l'âge de 18 mois, c'est à l'inverse le groupe I qui est favorisé (saison des pluies).

Les courbes de croissances en groupant ainsi les animaux par trimestre de naissances, s'entrecroisent en gardant une même allure générale. Il semble que les naissances en juin, juillet, août gardent un léger avantage, mais il est difficile d'être affirmatif.

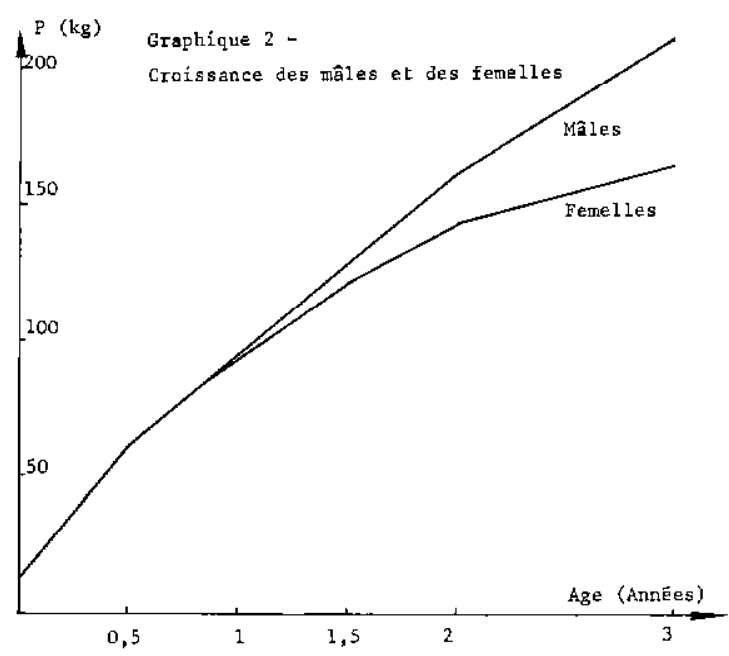




\section{Aptitude à l'embouche}

\section{Embouche sur Stylosanthes}

40 taurillons Baoulé nés à la Station de Bouaké ont été repris par le Centre de recherches zootechniques, afin d'étudier leur réaction à une embouche herbagère suivie d'une finition intensive (3). Leurs poids et âges moyens étaient de $148 \mathrm{~kg}$ et 22 mois.

Pendant une année entière, ces animaux ont été placés sur un pâturage de Stylosanthes guianensis à raison de 0,37 ha par tête, ce qui correspond à un chargement légèrement inférieur à 3 têtes/hectare ou à $450 \mathrm{~kg}$ de poids vif par hectare, en début d'essai. (Sous ce dernier aspect, la charge a augmenté en cours d'essai pour atteindre $650 \mathrm{~kg} / \mathrm{ha}$ in fine en raison de la croissance des animaux). Le pâturage réparti en quatre parcelles était utilisé par rotation.

L'évolution des poids et des gains est rapportée au tableau IV. une pointe en août (406 g/jour). L'année se termine avec des gains modestes dès que la saison sèche apparaît. (110 et $115 \mathrm{~g} /$ jour en novembre et décembre).

L'embouche au pâturage artificiel, même de légumineuses, ne permet pas d'obtenir des gains substantiels et de finir les animaux. C'est pourquoi l'emploi de concentrés a été pratiqué : sur 12 animaux des 40 précédents en complément du pâturage, sur 12 autres, de même origine, en zéro grazing dans un parc d'embouche.

12 autres animaux, enfin, restèrent sur Stylosanthes.

\section{Finition sur Stylosanthes avec complémentation}

Le concentré était constitué de graines de coton mélassées, puis de graines de coton et de farine basse de riz, en mélange.

Les taurillons demeurant au pâturage de Stylosanthes n'ont eu aucun problème d'adaptation.

TABL. ${ }^{\circ}$ IV-Embauche de 40 taurillons sur Stylosanthes guianensis

\begin{tabular}{|c|c|c|c|c|c|c|}
\hline & Dates & $\begin{array}{l}\text { Polds du lot } \\
\text { (kg) }\end{array}$ & $\begin{array}{l}\text { Gain par } \\
\text { période (kg) }\end{array}$ & G.Q.M. (g) & $\begin{array}{l}\text { Gain total } \\
(\mathrm{kg})\end{array}$ & G.Q.M. cumulatif \\
\hline $\begin{array}{r}8 \\
4 \\
27\end{array}$ & $\begin{array}{l}\text { décembre } \\
\text { janvier } \\
\text { février }\end{array}$ & $\begin{array}{ll}5 & 920 \\
6 & 211 \\
6 & 137\end{array}$ & $\begin{array}{l}291 \\
-74\end{array}$ & $\begin{array}{r}269,4 \\
-\quad 34,2\end{array}$ & $\begin{array}{l}291 \\
217\end{array}$ & $\frac{269,4}{67}$ \\
\hline $\begin{array}{l}31 \\
28\end{array}$ & $\begin{array}{l}\text { mars } \\
\text { avril }\end{array}$ & $\begin{array}{ll}6 & 392 \\
6 & 714\end{array}$ & $\begin{array}{l}255 \\
322\end{array}$ & $\begin{array}{l}199,2 \\
287,5\end{array}$ & $\begin{array}{l}472 \\
794\end{array}$ & $\begin{array}{l}104,4 \\
140,7\end{array}$ \\
\hline & $\begin{array}{l}\operatorname{mai} \\
\text { juin }\end{array}$ & $\begin{array}{ll}7 & 198 \\
7 & 316\end{array}$ & $\begin{array}{l}484 \\
118\end{array}$ & $\begin{array}{r}448,1 \\
84,3\end{array}$ & $\begin{array}{ll}1 & 278 \\
1 & 396\end{array}$ & $\begin{array}{l}190,1 \\
171,9\end{array}$ \\
\hline & $\begin{array}{l}\text { juillet } \\
\text { août }\end{array}$ & $\begin{array}{ll}7 & 576 \\
8 & 015\end{array}$ & $\begin{array}{l}260 \\
439\end{array}$ & $\begin{array}{l}224,1 \\
406,5\end{array}$ & $\begin{array}{ll}1 & 656 \\
2 & 095\end{array}$ & $\begin{array}{l}178,4 \\
202,2\end{array}$ \\
\hline & $\begin{array}{l}\text { septembre } \\
\text { octobre }\end{array}$ & $\begin{array}{ll}8 & 268 \\
8 & 459\end{array}$ & $\begin{array}{l}253 \\
191\end{array}$ & $\begin{array}{l}225,8 \\
164,6\end{array}$ & $\begin{array}{ll}2 & 348 \\
2 & 539\end{array}$ & $\begin{array}{l}204,5 \\
200,9\end{array}$ \\
\hline $\begin{array}{l}17 r \\
150\end{array}$ & $\begin{array}{l}\text { novembre } \\
\text { décembre }\end{array}$ & $\begin{array}{ll}8 & 578 \\
8 & 702\end{array}$ & $\begin{array}{l}119 \\
124\end{array}$ & $\begin{array}{l}110,2 \\
114,8\end{array}$ & $\begin{array}{ll}2 & 658 \\
2 & 782\end{array}$ & $\begin{array}{l}193,7 \\
187,9\end{array}$ \\
\hline
\end{tabular}

Les animaux prennent davantage de poids qu'au pâturage naturel: $69 \mathrm{~kg}$ en un an au lieu de 51 , mais le résultat demeure bien faible avec des gains quotidiens moyens inférieurs à $200 \mathrm{~g}$, c'est-à-dire voisins de $100 \mathrm{~g}$ par $100 \mathrm{~kg}$ de poids vif.

L'évolution des gains quotidiens montre l'influence considérable de la saison: en janvier et février, fin de la saison sèche, les taurillons ne prennent rien et même perdent légèrement. Puis on assiste à des gains quotidiens qui vont en s'accélérant avec la croissance de l'herbe, pour atteindre $448 \mathrm{~g}$ en mai. Après quoi, l'animal se stabilise un peu pour reprendre activement du poids en juillet, août et septembre avec
La durée de l'essai n'a pas été la même pour tous les animaux, car certains ont atteint assez vite un poids de $270 \mathrm{~kg}$ avec un bon état général, ce qui a conduit à les abattre plus tôt. Trois animaux ont été abattus après 53 jours, deux après 81 jours, 4 après 95 jours, 3 enfin après 103 jours. Pour l'ensemble, 1010 journées d'embouche ont entraîné un gain global de $457 \mathrm{~kg}$, soit un croît quotidien moyen de 452 grammes. Les résultats sont donnés au tableau V. On voit que le concentré a donné d'emblée des résultats spectaculaires (523 et $502 \mathrm{~g} / \mathrm{j})$ car les animaux maintenus sur leur pâturage n'ont pas eu de problème d'adaptation. Mais au cours du troisième mois les gains ont 
TABL. $N^{\circ} \mathrm{V}$-Pâturage complëmenté.

\begin{tabular}{|l|c|c|c|c|}
\hline Dates & Nombre & Poids (kg) & $\begin{array}{c}\text { Gain } \\
(\mathrm{kg})\end{array}$ & $\begin{array}{c}\text { G.Q.M. } \\
(\mathrm{g})\end{array}$ \\
\hline 15 décembre & 12 & 2682 & & \\
\hline 12 janvier & 12 & 2858 & 176 & 523 \\
\hline 14 février & 12 & 3057 & 199 & 502 \\
\cline { 2 - 5 } & 9 & 2229 & & \\
\hline \multirow{2}{*}{14 mars } & 9 & 2281 & 52 & 206 \\
\cline { 2 - 6 } & 7 & 1738 & & \\
\hline 28 mars & 7 & 1778 & 40 & 408 \\
\hline
\end{tabular}

nettement baissé (206 g) pour reprendre un peu sur les animaux les plus jeunes et les plus légers.

\section{Finition en parc}

Les douze taurillons mis en parc ont eu un comportement où l'agitation a été exacerbée par la promiscuité. Il en est résulté une adaptation assez difficile. Il ne paraît pas recommandẻ de placer en parc des mâles pubères.

Deux animaux ont été abattus après 53 jours, deux après 81 jours, deux autres après 88 jours, six après 103 jours.

Pour un total de 1062 jours de présence au parc, le gain total était de $345 \mathrm{~kg}$, soit $324 \mathrm{~g} /$ jour/tête. Le détail est donné au tableau VI.

TABL. $\mathrm{N}^{\circ} \mathrm{VI}-\mathrm{Finition}$ en parc.

\begin{tabular}{|c|c|c|c|c|}
\hline Dates & Nombre & Poids (kg) & $\begin{array}{c}\text { Gain } \\
(\mathrm{kg})\end{array}$ & $\begin{array}{c}\text { G.Q.M. } \\
(\mathrm{g})\end{array}$ \\
\hline 15 décembre & 12 & 2661 & & \\
\hline 12 janvier & 12 & 2745 & 84 & 250 \\
\hline \multirow{2}{*}{14 fêvrier } & 12 & 2895 & 150 & 378 \\
\cline { 2 - 6 } & 10 & 2358 & & \\
\hline \multirow{2}{*}{14 mars } & 10 & 2465 & 107 & 382 \\
\cline { 2 - 6 } & 6 & 1424 & & \\
\hline 28 mars & 6 & 1428 & 4 & 47 \\
\hline
\end{tabular}

Les gains quotidiens sont moins élevés qu'au pâturage supplémenté mais ils se poursuivent mieux au cours du troisième mois pour s'effondrer en fin d'essai ( $47 \mathrm{~g}$ / jour).
Les aliments, entièrement distribués à l'auge, ont pu être mesurés :

Fourrage : $17946 \mathrm{~kg}$ soit 1345 U.F.

Concentré : $3009 \mathrm{~kg}$ soit 3097 U.F.

Total

4442 U.F.

Le gain total étant de $345 \mathrm{~kg}$, l'indice de consommation s'est élevé à $4442=12,87$ U.F. 345

par $\mathrm{kg}$ de gain, ce qui est trop élevé pour permettre l'économie de l'opération.

\section{Lot témoin}

Le lot témoin sur Stylosanthes, pendant le même temps, a pris $321 \mathrm{~kg}$ en 1496 jours de pâturage effectif, soit $214 \mathrm{~g}$ /jour. On retrouve une perte de poids en mars, qui correspond au passage de la fin de la saison sèche au début des pluies, phénomène très classique.

Le tableau VII retrace l'évolution des poids du lot, dont les animaux ont été, ici aussi, abattus de façon échelonnée.

TABL. $N^{0}$ VII-Témoins au pâturage.

\begin{tabular}{|c|c|c|c|c|}
\hline Dates & Nombre & Poids $(\mathrm{kg})$ & $\begin{array}{c}\text { Gain } \\
(\mathrm{kg})\end{array}$ & $\begin{array}{c}\text { G.Q.M. } \\
(\mathrm{g})\end{array}$ \\
\hline 15 décembre & 16 & 3359 & & \\
\hline 12 janvier & 16 & 3511 & 152 & 339 \\
\hline \multirow{2}{*}{14 février } & 16 & 3647 & 136 & 257 \\
\cline { 2 - 5 } & 14 & 3098 & & \\
\hline \multirow{2}{*}{14 mars } & 14 & 3144 & 46 & 117 \\
\cline { 2 - 6 } & 11 & 2363 & & \\
\hline 28 mars & 11 & 2350 & -13 & -84 \\
\hline
\end{tabular}

\section{Conclusion}

En finition, le Baoulé donne des résultats qui peuvent paraître modestes : $500 \mathrm{~g}$ par jour pendant deux mois, $400 \mathrm{~g}$ pendant une période légèrement plus longue. Mais si l'on rapporte ces gains au poids vif de l'animal, ils sont de l'ordre de $200 \mathrm{~g}$ par $100 \mathrm{~kg}$ /vif ce qui, sans être très brillant, apparaît moins faible.

Si l'on veut des carcasses pesant plus de $100 \mathrm{~kg}$, le format de l'animal étant petit, on est obligé d'emboucher des animaux déjà pubères.

A ce stade, le pouvoir transformateur des aliments est moins bon. 


\section{Production de viande}

En Côte-d'Ivoire, l'on abat des zébus provenant du Sahel dont les carcasses peuvent dépasser $200 \mathrm{~kg}$; les carcasses de N'Dama atteignent fréquemment $150 \mathrm{~kg}$. Cela fait que lès carcasses de Baoulé qui n'atteignent pas toujours $100 \mathrm{~kg}$, sont moins appréciées. Le prix obtenu par $\mathrm{kg}$ est toujours un peu inférieur à celui des races citées précédemment.

La qualité de la viande est diversement appréciée. Pour les uns excellente, pour les autres fibreuse et peu sapide.

L'analyse d'une carcasse de l'un des animaux finis au cours des essais précédents a donné les résultats suivants :

\begin{tabular}{|c|c|c|}
\hline \multirow{2}{*}{\multicolumn{2}{|c|}{$\begin{array}{l}\text { Poids vif au départ du Centre } \\
\text { Poids vif après } 24 \text { heures de jeûne (1) }\end{array}$}} & \\
\hline & & $249 \mathrm{~kg}$ \\
\hline Poids du contenu digestif & & $25 \mathrm{~kg}$ \\
\hline ide (2) & & $224 \mathrm{~kg}$ \\
\hline de carcasse chaude (3) & & $135 \mathrm{~kg}$ \\
\hline ement commercial $(3) /(1)$ & & 54,8 p. 100 \\
\hline t vrai $(3) /(2)$ & * & o. 100 \\
\hline casse (4) & . & $98 \mathrm{~cm}$ \\
\hline$(3) /(4)$ & . & 1,37 \\
\hline se (5). & & $19,2 \mathrm{~cm}$ \\
\hline (1) & & \\
\hline e rognons $(6)$ & & $\mathrm{kg}$ \\
\hline $\operatorname{ras}(6) /(3)$ & & \\
\hline
\end{tabular}

On voit que la carcasse outre sa petite taille et son poids modeste, bien qu'il s'agisse d'un animal fini, se présente comme assez peu musclée. Les animaux à viande (Charolais) ont des indices de muscle atteignant 0,28. Avec 0,19, le Baoulé se retrouve au niveau du zébu dont la conformation musculaire est plutôt plate.

En second lieu, il est surprenant de trouver sur un animal ayant été fini, un indice de gras aussi faible que 0,5 p. 100 ; on s'attendrait à un indice voisin de 2 ou supérieur. Bien sûr. il s'agit d'animaux entiers, mais ce résultat confirme l'opinion générale qui veut que le Baoulé se finisse mal avec dépôts de graisse insuffisants. Cela est sans aucun doute un facteur défavorable à la sapidité de la viande.

\section{Production de lait}

Nous n'avons connaissance d'aucun contrôle de production laitière de vaches Baoulé. On peut tenter d'approcher la production laitière par la croissance des veaux à la mamelle.

Si l'on applique la formule :

$$
\mathrm{Q}=9,18 \frac{\mathrm{P} 4-\mathbf{P n}}{120}
$$

où $\mathbf{P} 4=$ poids à 4 mois, $\mathrm{Pn}=$ poids de naissance on obtient avec les animaux de la station (185 veaux et velles)

$\mathrm{Q}=9,18 \times \frac{45,9-12,25}{120}=2,5741 /$ jour

ou 308,9 litres en quatre mois ce qui est, évidemment, très faible.

Dans les élevages traditionnels, après avoir séparé et attaché les veaux pendant la nuit, les bergers Peuls traient les vaches Baoulé avant de les conduire au pâturage. La quantité prélevée est toujours modeste et il faut de nombreuses vaches pour que le $P$ eul puisse vendre quelques litres de lait.

Compte tenu cependant de la faible production des vaches, la traite devient catastrophique pour les veaux et l'on ne peut plus s'étonner de ce que, dans l'élevage confié aux Peuls, un veau sur deux puisse mourir.

\section{PATHOLOGIE}

Les mortalités, de très loin les plus importantes, sont à déplorer sur les veaux. Sur 438 veaux nés, 61 (14 p. 100) sont morts dont :

25 avant 15 jours,

26 entre 15 jours et 3 mois,

4 entre 3 mois et 9 mois,

6 entre 9 mois et 12 mois.

Les causes essentielles de mortalité avant 3 mois se répartissent comme suit :

Poids insuffisant à la naissance . . . 4

Diarrhées . . . . . . . . . . . 24

Lactation insuffisante de la mère . . . 9

Il demeure, comme dans tout élevage extensif, un certain nombre de mortalités auxquelles on ri'a pas pu trouver une cause précise (le plus souvent à cause d'une autopsie tardive et d'un cadavre en mauvais état de conservation).

Le facteur alimentaire paraît primordial, car de nombreux veaux sont insuffisamment nourris par leur mère, surtout par les primipares ce qui dıminue leur résistance, favorise les déviations des populations microbiennes du tube digestif et les infections fatales.

Il faut par conséquent s'attacher à mieux nourrir les vaches en toutes saisons pour qu'elles nourrissent mieux leurs veaux; toute améliora- 
tion génétique devrait s'efforcer d'introduire (modérément bien sûr) quelques gènes laitiers, afin que l'herbe soit mieux utilisée.

Sur les adultes, on a relevé un cas de météorisation, un cas d'intoxication, un accident; mais la mortalité, à ce stade, demeure très faible.

Il faut surtout retenir que l'action sanitaire doit prioritairement porter sur les jeunes si l'on veut augmenter la productivité du troupeau.

\section{CONCLUSION GENERALE}

La race Baoulé apparaît au premier regard comme peu productive: format très faible, croissance lente, engraissement insuffisant, lactation très déficiente. Cependant, il convient de bien examiner les qualités dont elle est dotée; elles expliquent parfaitement sa diffusion dans les savanes humides proches de la forêt :

- la tolérance à la trypanosomiase lui permet de subsister sans dommage dans les régions infestées par les glossines;

- la résistance aux maladies transmises par les tiques est également très bonne;
- l'adaptation au climat tropical humide est excellente : les vaches présentent une fécondité élevée, ainsi qu'une précocité indéniable.

Ces qualités sont donc à prendre en compte par tout programme d'amélioration de la production bovine dans les régions de savanes humides de l'Afrique de l'Ouest.

Si la sélection ne peut pas laisser espérer un accroissement suffisant de la productivité de la race Baoulé, les zootechniciens seront vraisemblablement amenés à étudier des croisements. Ils devront être entrepris avec prudence et discernement. Il est sans doute nécessaire d'accroitre le format des animaux de manière à pouvoir les utiliser en boucherie à un âge suffisamment peu élevé : on bénéficiera alors de leur meilleur pouvoir transformateur de la nourriture tant qu'ils sont jeunes. Mais il faut se rappeler que le marché ne réclame pas des carcasses très lourdes. Les infusions de gènes améliorateurs devront rester limitées de façon à conserver, dans la proportion la plus forte possible, le potentiel génétique de résistance aux maladies, d'adaptation au milieu, en un mot de rusticité, de la race Baoulé.

\section{SUMMARY}

\section{Study of a Baoule cattle breed in Ivory Coast}

Baoulé cattle, a trypanotolerant breed, is the most numerous in Ivory Coast. A herd was controlled at Bouake husbandry center (sudanoguinean savannah).

Baoule cattle is a small breed: height at withers $100 \mathrm{~cm}$ for bulls and $95 \mathrm{~cm}$ for cows. But the breed is very well adapted to the climate and environment: high fertility, precocious first calving and good resistance to diseases.

The growing characteristics were studied. Fattening on Stylosanthes guianensis pasture or with concentrate diets given a fast growth. Carcasses are light with few fat.

The scour of calves causes a high percentage of death.

\section{RESUMEN}

\section{Estudio de una poblacion de bovinos Baule en Costa de Marfil}

Los bovinos Baule, resistentes contra la tripanosomiasis, son más numerosos en Costa de Marfil.

En Buake (region de sabana sudano gunéana) se ha criado y comprobado una manada.

El Baule es una pequeña raza : machos: $100 \mathrm{~cm}$ a la cruz, hembras $95 \mathrm{~cm}$. Pero la raza muestra su adaptación al medio por una excelente fertilidad, una edad al primer parto muy precoz y una buena resistencia contra las enfermedades. Se estudian las características del crecimiento.

El acabado sea sobre pasto de Stylosanthes guianensis, sea con un pienso concentrado permite obtener un crecimiento más rapido.

Los canales son ligeros y poco grasos. La diarrea de los terneros causa las mortalidades más importantes. 


\section{BIBLIOGRAPHIE}

1. AILLERIE. Rapports au Gouverneur de Bingerville; 1923, 1924, 1925, 1933. Arch. Nat. (Cốted'Ivoire). Dossiers VI - 16-244, VI - 16-237, VI 4-159.

2. DOUTRESSOULLE (G.). L'élevage en Afrique occidentale française. Paris, Larose, 1947, 298 p.

3. GOMBAUD (B.). Embouche de taurins Baoulé et N'Dama en zone de savane. Bouaké-Minankro, C.R.Z. I.E.M.V.T., République de Côte-d'Ivoire, Ministère de la Recherche Scientifique, octobre 1973, 63 p. (rap. ronéo.).

4. JOSHI (N. R.), Mc LAUGHLIN (E. A.), PHIL-
LIPS (R. W.). Les bovins d'Afrique, types et races. Rome, F.A.O., 1957, 317 p. (Etude agricole de la F.A.O. $n^{\circ}$ 37)

5. PAGOT (J.), COULOMB (J.), PETIT (J. P.). Revue et situation actuelle de l'emploi des races trypanotolérantes. Bull. Inf. tech. trypanosomiases africaines. O.M.S./F.A.O./Try./Inf./74.57.

6. VERLY (P. L.). Contribution à l'étude des races bovines autochtones en Côte-d'Ivoire: les bœufs Baoulé et Lagunes. D.E.S. Université Abidjan, Fac. Sci., juin 1968, 213 p. 